IJMMS 30:10 (2002) 621-625

PII. S0161171202007603

http://ijmms.hindawi.com

(c) Hindawi Publishing Corp.

\title{
THE UNION PROBLEM ON COMPLEX MANIFOLDS
}

\author{
PATRICK W. DARKO
}

Received 14 May 2001

Let $\Omega$ be a relatively compact subdomain of a complex manifold, exhaustable by Stein open sets. We give a necessary and sufficient condition for $\Omega$ to be Stein, in terms of $L^{2}$-estimates for the $\bar{\partial}$-operator, equivalent to the condition of Markoe (1977) and Silva (1978).

2000 Mathematics Subject Classification: 32E10, 32C35, 35N15.

1. Introduction. As indicated in [7], from the beginning of the theory of Stein spaces, the following question has held great interest: is a complex space, which is exhaustable by a sequence $X_{1} \Subset X_{2} \Subset \cdots$ of Stein subspaces, itself Stein?

In [1], the following is proved: every domain in $\mathbb{C}^{m}$ which is exhaustable by a sequence of Stein domains $B_{1} \Subset B_{2} \Subset \cdots$ is itself Stein, and this is shown to hold more generally for unramified Riemann domain $\mathscr{B}$ over $\mathbb{C}^{m}$ in [6]. In [11], the following is proved: let $X$ be a reduced complex space and $X_{1} \Subset X_{2} \Subset \cdots$ be an exhaustion of $X$ by Stein domains, if every pair $\left(X_{j}, X_{j+1}\right)$ is Runge then $X=U X_{j}$ is Stein. Recently, Markoe [9] and Silva [10] proved the following: let $X$ be reduced and $X_{1} \Subset X_{2} \Subset \ldots$ be an exhaustion of $X$ by Stein domains. Then $X$ is Stein if and only if $H^{1}(X, \mathcal{O})=0$ ( $O$ being the structure sheaf of $X$ ).

More recently the following has been proved in [12]: let $\Omega_{1} \subset \Omega_{2} \subset \cdots$ be a sequence of open Stein subsets of a Stein space $X, \Omega=\bigcup_{j=1}^{\infty} \Omega_{j}$, and $\operatorname{dim} H^{1}(\Omega, \mathcal{O})<\infty$. Then $\Omega$ is Stein.

Fornæss [4] produced an example to show that if $X_{1} \Subset X_{2} \Subset \cdots$ is a sequence of Stein manifolds, the limit manifold $X=\bigcup X_{j}$, in which each $X_{j}$ is an open submanifold, need not be Stein. But it is known that if the limit manifold is itself an open submanifold of a Stein manifold then the limit manifold is necessarily Stein.

This led Fornæss and Narasimhan to pose the following problem [5]: let $X$ be a Stein space and $\Omega_{1} \Subset \Omega_{2} \Subset \cdots$ an increasing sequence of Stein open sets in $X$. Is $\bigcup \Omega_{j}$ Stein? As indicated above this is the case when $X$ is a Stein manifold, but this question remains open in the general case.

In this paper, we consider the case where $X$ is a general complex manifold and $\Omega_{1} \Subset$ $\Omega_{2} \Subset \cdots$ an increasing sequence of open Stein manifolds in $X$ such that $\Omega=\bigcup \Omega_{j}$ is relatively compact in $X$. We give a condition for $\Omega$ to be Stein, equivalent to Markoe's and Silva's condition and involving $L^{2}$-estimates for the $\bar{\partial}$ operator.

2. Preliminaries. Let $X$ be an $n$-dimensional complex manifold with a $C^{\infty}$ Hermitian metric. The space $L_{(p, q)}^{2}(X)$ of square integrable differential forms of type $(p, q)$ on $X$ 
is a Hilbert space under the scalar product,

$$
(f, g)=\int_{X} f_{\wedge} * \bar{g}
$$

where $*$ is the Hodge $*$-operator associated with the metric and orientation of $X$.

Let $\Omega_{1} \Subset \Omega_{2} \Subset \cdots$ be an increasing sequence of Stein open sets in $X$ such that their union $\Omega=\bigcup_{j=1}^{\infty} \Omega_{j}$ is relatively compact in $X$.

The following theorem is our main result.

THEOREM 2.1. The union $\Omega$ is Stein if and only if given an $f \in L_{(p, q)}^{2}(\Omega)$, which is $\bar{\partial}$-closed in the sense of distributions, there is a $u \in L_{(p, q-1)}^{2}(\Omega)$ such that $\bar{\partial} u=f$ in the sense of distributions and

$$
\|u\|_{L^{2}(p, q-1)} \leq K\|f\|_{L^{2}(p, q)(\Omega)}, \quad q>0,
$$

where K depends on $\Omega$.

Let $U$ be a bounded open set in $\mathbb{C}^{n}$, and 0 the structure sheaf of $\mathbb{C}^{n}$. A section $f=\left(f_{1}, \ldots, f_{p}\right) \in \Gamma\left(U, \mathcal{O}^{p}\right)$, where $p>0$ is an integer, is $L^{2}$-bounded if

$$
\|f\|_{L^{2}(U)}=\left\|f_{1}\right\|_{L^{2}(U)}+\cdots+b\left\|f_{p}\right\|_{L^{2}(U)}<\infty .
$$

We then denote all sections of $\mathcal{O}^{p}$ over $U$ that are $L^{2}$-bounded by $\Gamma_{2}\left(U, \mathscr{O}^{p}\right)$.

For the definition of $L^{2}$-bounded sections of coherent analytic sheaves, we require the coherent analytic sheaf $\mathscr{F}$ to be defined on a simply connected polycylinder neighborhood $V$ of the closure of $U$. Then by [8, Theorem 5, Section F, Chapter VI], there is an $\mathbb{O}$-homographic in another simply connected polycylinder neighborhood $V^{\prime}$ of the closure of $U$,

$$
\mathrm{O}^{p} \stackrel{\lambda}{\longrightarrow} \mathscr{F} \longrightarrow 0,
$$

where $p>0$ is some integer; and $f \in \Gamma(U, \mathscr{F})$ is $L^{2}$-bounded if $f \in \Gamma_{2}(U, \mathscr{F}):=\lambda\left(\Gamma_{2}(U\right.$, $\left.\left.\mathcal{O}^{p}\right)\right)$. It can be shown that $\Gamma_{2}(U, \mathscr{F})$ is independent of $\lambda$ and $p$, so that $\Gamma_{2}(U, \mathscr{F})$ is well defined.

Now let $\Omega$ be a relatively compact subdomain of an $n$-dimensional complex manifold $X$. An open subset $Y$ of $\Omega$ is said to be admissible for the coherent analytic sheaf $\mathscr{F}$ defined in the neighborhood of the closure of $\Omega$ in $X$, if $Y$ is Stein. There is a coordinate neighborhood $V$ in $X$ of the closure, $\bar{Y}$ of $Y$ such that $V$ is biholomorphic to a simply connected polycylinder $V^{\prime}$ in $\mathbb{C}^{n}$, and $\bar{Y}$ is contained in the neighborhood of $\bar{\Omega}$ where $\mathscr{F}$ is defined as $f \in \Gamma(Y, \mathscr{F})$ which is $L^{2}$-bounded if

$$
f \in \Gamma_{2}(Y, \mathscr{F}):=\left\{g \in \Gamma(Y, \mathscr{F}): \eta_{*}(g) \in \Gamma_{2}\left(\eta(Y), \eta_{*}(\mathscr{F})\right)\right\},
$$

where $\eta$ is the restriction of the biholomorphic map $V \rightarrow V^{1}$ to $Y$, and $\eta_{*}(\mathscr{F})$ is the zero direct image of $\mathscr{F}$ on $Y$.

Let $\Omega$ be as in Theorem 2.1 (then clearly $\Omega$ is locally Stein). Let $\mathscr{F}$ be a coherent analytic sheaf in a neighborhood of the closure of $\Omega$. Then it is clear that $\Omega$ is a finite union, $\Omega=\bigcup_{j=1}^{m} U_{j}$, where each $U_{j}$ is admissible for $\mathscr{F}$. If $\mathscr{V}=\left\{U_{j}\right\}_{j \in I}, I=\{1, \ldots, m\}$, 
where the $U_{j}$ 's are as above, we say that $\mathscr{V}$ is a finite admissible cover of $\Omega$ for $\mathscr{F}$ and we define the $L^{2}$ (alternate) $q$-cochains of $\mathscr{V}$ with values in $\mathscr{F}$ as those cochains,

$$
\begin{gathered}
c=\left(c_{\alpha}\right) \in C^{q}(\mathscr{V}, \mathscr{F})=\prod_{\alpha \in I^{q+1}} \Gamma\left(U_{\alpha}, \mathscr{F}\right), \\
U_{\alpha}=U_{i_{0}} \cap \cdots \cap U_{i_{q}}, \quad \alpha=\left(i_{0}, \ldots, i_{q}\right),
\end{gathered}
$$

which are alternate and satisfy $c_{\alpha} \in \Gamma_{2}\left(U_{\alpha}, \mathscr{F}\right)$ for all $\alpha \in I^{q+1}$. We denote by $C_{2}^{q}(\mathscr{V}, \mathscr{F})$ the space of $L^{2}$-bounded cochains.

The coboundary operator,

$$
\delta: C^{q}(\mathscr{V}, \mathscr{F}) \rightarrow C^{q+1}(\mathscr{V}, \mathscr{F}),
$$

maps $C_{2}^{q}(\mathscr{V}, \mathscr{F})$ into $C_{2}^{q+1}(\mathscr{V}, \mathscr{F})$. If $Z_{2}^{q}(\mathscr{V}, \mathscr{F})=\left\{c \in C_{2}^{q}(\mathscr{V}, \mathscr{F}): \delta c=0\right\}$ and $B_{2}^{q}(\mathscr{V}, \mathscr{F})=$ $\delta C_{2}^{q-1}(\mathscr{V}, \mathscr{F})$, then as usual $B_{2}^{q}(\mathscr{V}, \mathscr{F}) \subseteq Z_{2}^{q}(\mathscr{V}, \mathscr{F})$ and we define $H_{2}^{q}(\mathscr{V}, \mathscr{F}):=Z_{2}^{q}(\mathscr{V}, \mathscr{F}) /$ $B_{2}^{q}(\mathscr{V}, \mathscr{F})$ and call it the $L^{2}$-bounded cohomology of $\mathscr{V}$ with values in $\mathscr{F}$. We then have the following theorem.

THEOREM 2.2. For any $q>0$, the natural map

$$
H_{2}^{q}(\mathscr{V}, \mathscr{F}) \longrightarrow H^{q}(\Omega, \mathscr{F})
$$

is an isomorphism.

We use Theorem 2.2 as a pivot to prove Theorem 2.1, but the proof of Theorem 2.2 is not given here, since it is similar to that of [2, Theorem].

3. A triangle of isomorphisms. Let $\Omega$ be as in Theorem 2.1. By the end of the section Theorem 2.1 will be proved. If $U \neq \varnothing$ is an open set in $\bar{\Omega}$, then $\mathscr{P}_{\Omega}^{p}(U)$ is the Hilbert space of holomorphic $p$-forms $h$ on $\Omega \cap U$ such that

$$
\|h\|_{L_{(p, 0)(\Omega \cap U)}^{2}}<\infty .
$$

If $V$ is open in $\bar{\Omega}$ with $\varnothing \neq V \subset U$, the restriction map $\gamma_{V}^{U}: \mathscr{P}_{\Omega}^{p}(U) \rightarrow \mathscr{P}_{\Omega}^{p}(V)$ is defined. Then $\mathscr{B}_{0}^{p}=\left\{\mathscr{B}_{\Omega}^{p}(U), \gamma_{V}^{U}\right\}$ is the canonical presheaf of $L^{2}$-holomorphic $p$-forms on $\bar{\Omega}$. The associated sheaf $\mathscr{R}_{2}^{p}$ is the sheaf of germs of $L^{2}$-holomorphic $p$-forms on $\bar{\Omega}$. We then have the following lemma.

LEMMA 3.1. Let $\mathscr{D}^{p}$ be the sheaf of germs of holomorphic $p$-forms on $X$, and $\mathscr{V} a$ finite admissible cover of $\Omega$ for $\mathscr{D}^{p}$. Then the following diagram is an isomorphism triangle of cohomology groups:

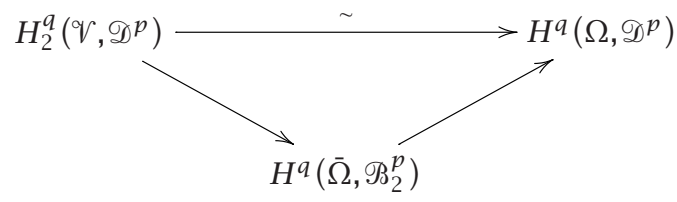

for $q \geq 1$ and $p \geq 0$. 
Proof. From Theorem 2.2 and the fact that any finite cover of $\bar{\Omega}$ has a refinement $\mathcal{U}=\left\{V_{j}\right\}_{j \in J}$ such that $\mathcal{U}_{\Omega}=\left\{V_{j} \cap \Omega\right\}_{j \in J}$ is a finite admissible cover of $\Omega$ for $\mathscr{D}^{p}$, the lemma follows.

Now, using Hörmander's $L^{2}$-estimates locally we get the following lemma.

LEMMA 3.2. The cohomology group $H^{q}\left(\bar{\Omega}, \mathscr{\Re}_{2}^{p}\right)$ is isomorphic to the quotient space

$$
\left\{g: g \in L_{(p, q)}^{2}(\Omega) \text { and } \bar{\partial} g=0\right\} /\left\{\bar{\partial} h: h \in L_{p, q-1}^{2}(\Omega) \text { and } \bar{\partial} h \in L_{(p, q)}^{2}(\Omega)\right\} \text {, }
$$

where $\Omega$ is as in Theorem 2.1 .

Also the following lemma is proved in [3].

LEMmA 3.3. If $\Omega \Subset X$ is Stein, where $X$ is a complex manifold, then given $f \in$ $L_{(p, q)}^{2}(\Omega)$ with $\bar{\partial} f=0$, there is $u \in L_{(p, q-1)}^{2}(\Omega)$ such that

$$
\bar{\partial} u=f, \quad\|u\|_{L^{2}(p, q-1)(\Omega)} \leq K\|f\|_{L^{2}(p, q)(\Omega)},
$$

where $K$ depends on $\Omega$.

To finish with the proof of Theorem 2.1 we remark that $\mathscr{D}^{0}=0$ is the structure sheaf of $X(X, \Omega$ as in Theorem 2.1), therefore Theorem 2.1 follows from Lemmas 3.1, 3.2, and 3.3, and from Markoe's and Silva's condition.

\section{REFERENCES}

[1] H. Behnke and K. Stein, Konvergente folge von regularit ätsbereichen und die meromorphiekonvexität, Math. Ann. 116 (1938), 204-216 (German).

[2] P. W. Darko, On cohomology with bounds on complex spaces, Atti Accad. Naz. Lincei Rend. Cl. Sci. Fis. Mat. Natur. (8) 60 (1976), no. 3, 189-194.

[3] _ $L^{2}$ estimates for the $\bar{\partial}$ operator on Stein manifolds, Math. Proc. Cambridge Philos. Soc. 129 (2000), no. 1, 73-76.

[4] J. E. Fornæss, An increasing sequence of Stein manifolds whose limit is not Stein, Math. Ann. 223 (1976), no. 3, 275-277.

[5] J. E. Fornæss and R. Narasimhan, The Levi problem on complex spaces with singularities, Math. Ann. 248 (1980), no. 1, 47-72.

[6] H. Grauert and R. Remmert, Konvexität in der komplexen Analysis. Nicht-holomorphkonvexe Holomorphiegebiete und Anwendungen auf die Abbildungstheorie, Comment. Math. Helv. 31 (1956), 152-160, 161-183 (German).

[7] _ Theory of Stein Spaces, Springer-Verlag, Berlin, 1979.

[8] R. C. Gunning and H. Rossi, Analytic Functions of Several Complex Variables, PrenticeHall, New Jersey, 1965.

[9] A. Markoe, Runge families and inductive limits of Stein spaces, Ann. Inst. Fourier (Grenoble) 27 (1977), no. 3, 117-127.

[10] A. Silva, Rungescher Satz and a condition for Steinness for the limit of an increasing sequence of Stein spaces, Ann. Inst. Fourier (Grenoble) 28 (1978), no. 2, 187-200 (German).

[11] K. Stein, Überlagerungen holomorph-vollständiger komplexer Räume, Arch. Math. 7 (1956), 354-361 (German). 
[12] L. M. Tovar, Open Stein subsets and domains of holomorphy in complex spaces, Topics in Several Complex Variables (Mexico, 1983), Pitman, Massachusetts, 1985, pp. 183189.

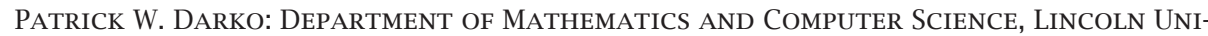
VERSITY, LINCOLN UNIVERSITY, PA 19352, USA

E-mail address: pdarko@7u.1incoln.edu 


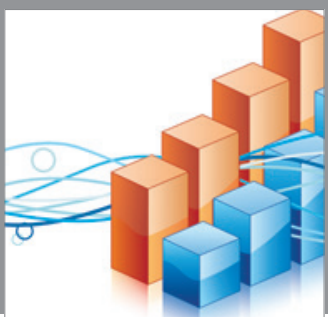

Advances in

Operations Research

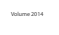

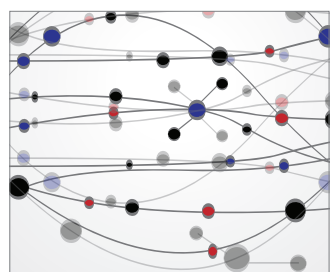

\section{The Scientific} World Journal
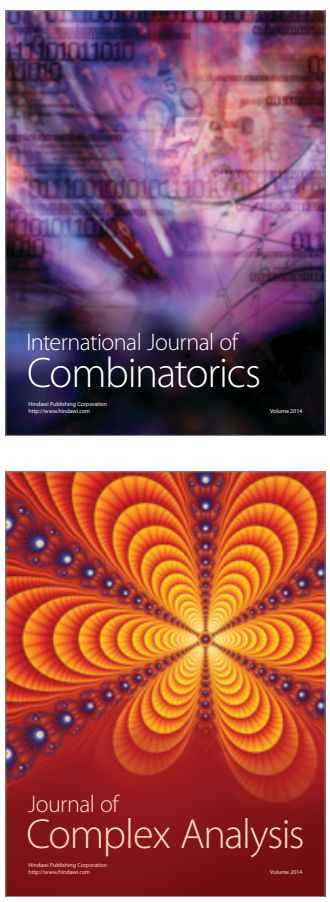

International Journal of

Mathematics and

Mathematical

Sciences
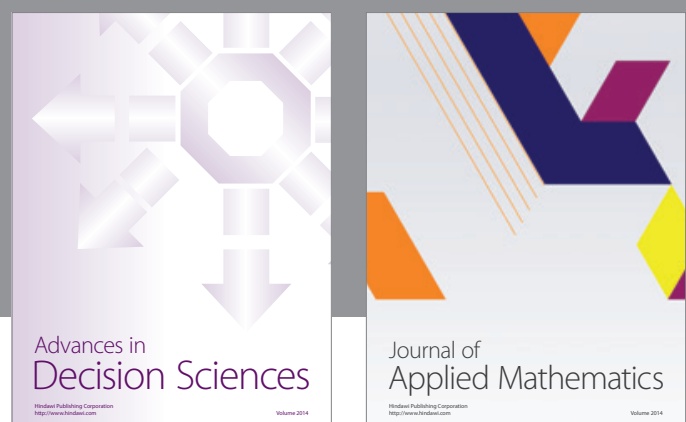

Journal of

Applied Mathematics
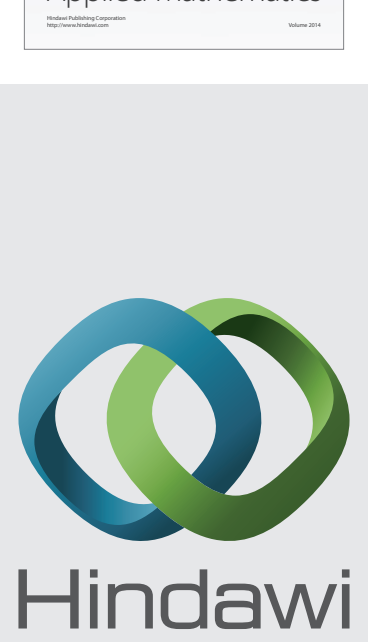

Submit your manuscripts at http://www.hindawi.com
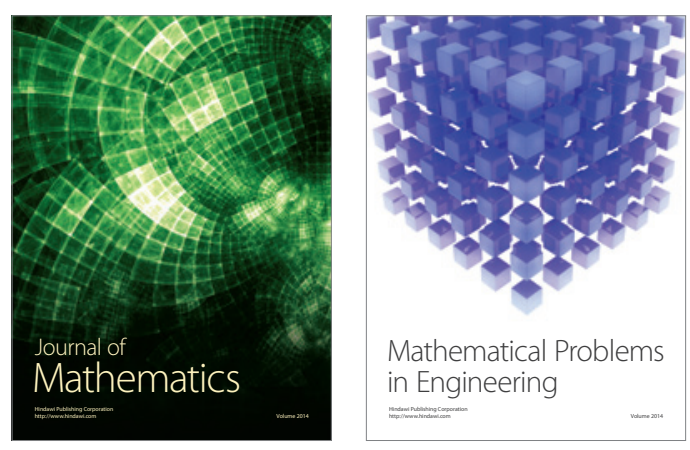

Mathematical Problems in Engineering
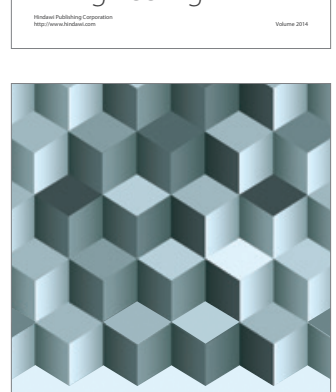

Journal of

Function Spaces
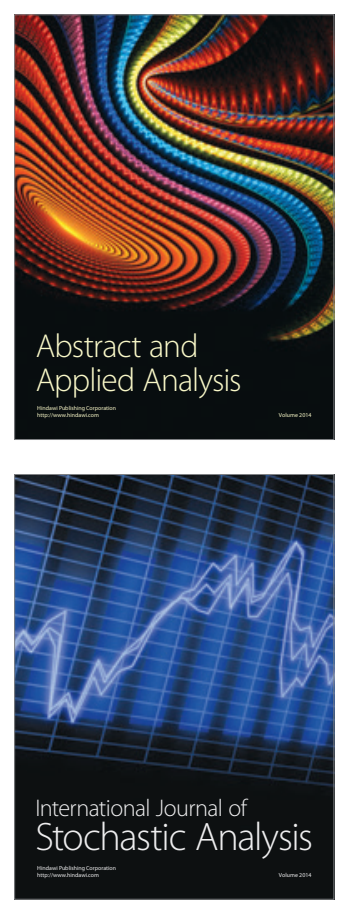

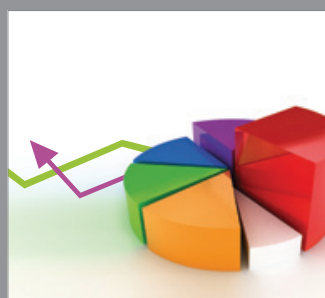

ournal of

Probability and Statistics

Promensencen
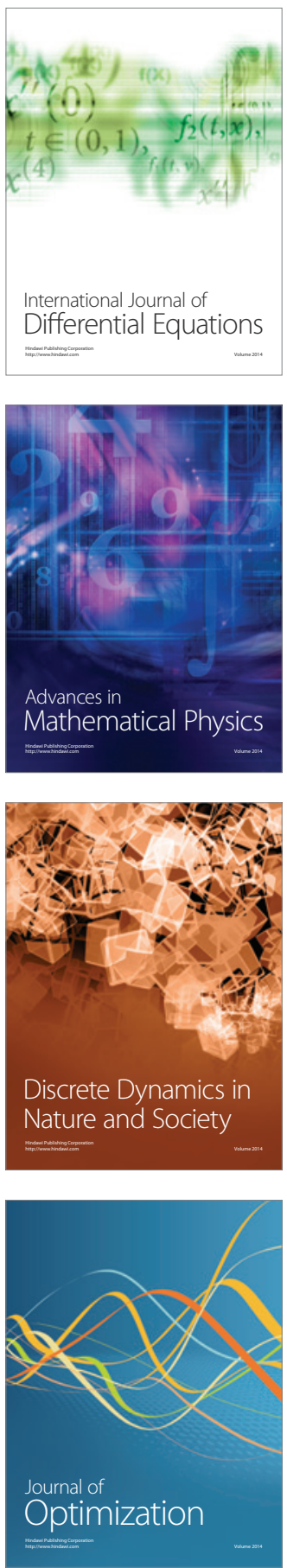